\title{
AURC from T1 to T2 Normalized by Body Mass Index
}

National Cancer Institute

\section{Source}

National Cancer Institute. AURC from T1 to T2 Normalized by Body Mass Index. NCI

Thesaurus. Code C92350.

The area under the urinary excretion rate curve (AURC) over the interval from T1 to T2 divided by the body mass index. 